\title{
High Frequency and High Power Density Transformers for DC/DC Converter used in Solar PV System
}

\author{
J. Lu*, S. Stegen*, and D. Butler** \\ * School of Engineering, Griffith University, Nathan, Brisbane, Qld 4111, Australia \\ ** Surtek Pty Ltd, Acacia Ridge, Brisbane, Qld 4110, Australia
}

\begin{abstract}
This paper presents and investigates planar and coaxial high frequency power transformers used for DC/DC converters in a three phase photo voltaic (PV) power systems. The winding structure including a Faraday shield between the primary and secondary windings is designed to minimize eddy current losses, skin and proximity effects, and to reduce the leakage inductance, and the inter winding coupling capacitance. Finite Element Method is employed to analyze the magnetic flux and eddy current distributions. The two different kinds of prototype high frequency transformers are designed and tested. The simulation and experiment results are demonstrated and compared with non-shielded transformers. The shielded transformers have achieved the expected results with a relatively small coupling capacitance, compared with the conventional high frequency transformer. This shield decreases the inter-winding coupling capacitance $C_{\mathrm{ps}}$.
\end{abstract}

Index Terms -- DC/DC converter, High frequency transformer, Solar PV system, Shielding.

\section{INTRODUCTION}

Photovoltaic arrays are coupled to the power grid using a DC-DC converter, a DC-AC inverter and an isolation transformer. The isolation transformer is typically large and bulky as the operating frequency is the utility frequency. A way of avoiding the use of a large transformer is to employ a DC-AC-DC (DC/DC) converter with a high frequency (HF) link isolation transformer, as shown in Fig. 1. Higher output power from multiple solar PV modules is achieved by connecting each PV array to its own full bridge DC/DC converter and single phase transformer. The topology chosen is for a 3-phase open delta to wye converter. The DC-AC converter output voltages are phase shifted with respect to each other by $120^{\circ}$. Overshoot and ringing on the primary side is almost completely avoided and there is a factor of 6 reduction in ripple current magnitude. This means a reduction of 6 in the size of both the output inductor and the input capacitor over the single phase alternative. The secondaries of the HF link transformers are connected in a three-phase wye configuration and the output is connected to a three phase rectifier. This configuration concept can be extended to $n$ phases. The $\mathrm{DC} / \mathrm{AC}$ inverter is used to transfer power to the utility and is synchronized to the utility frequency. The three full bridge converters are phase controlled to provide a minimal switching loss waveform. Voltage stresses are minimised and there is excellent current sharing among the active devices.

With a 1:1 transformer ratio there is a 1:2 voltage step up, this is advantageous PV array matching for maximum power transfer. The power converter architecture connected to the primary terminals of the transformers and the output rectifier can be either current source fed or voltage source. Inverters with isolation transformers are essential in many countries considering the safety and reliability of solar PV installations.

In this paper, high frequency planar and coaxial transformers [1-3], with and without Faraday shield, with an operating frequency up to $300 \mathrm{kHz}$, using a FEM based numerical modelling technique and high frequency measurement techniques are investigated. A number of issues related to magnetic flux, current distributions, power losses in windings and the Faraday shield implementation are discussed. Finally, the parasitic capacitances of the model are evaluated using a FEM based commercial available simulator (such as Ansys) and measurements for the intra- and inter- winding capacitances.

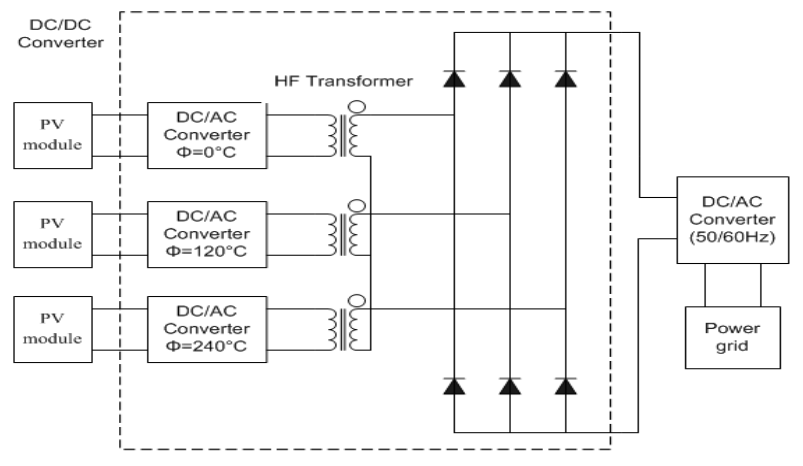

Fig.1. The three phase DC/DC converter for solar PV power conversion systems and the block diagram of PV system.

\section{Structure CONFIGURATIONS OF HF TRANSFORMERS}

The basic structure of the shielded HF coaxial transformer (HFCT) [4] is shown in Fig. 2. Surrounded by four round ferro-magnetic cores, the primary and the secondary winding are connected through the planar layers on the top and bottom (a). Inside the cores, between the primary and secondary side, the faraday shield is implemented with a copper cylinder (b). It can be seen, that there is a 30 degree offset between the turns. 
The planar transformer (step up) version as shown in Fig. 3 has a faraday shield copper coated layer between the primary and secondary side. With a $1: 1$ or $1: n$ turns ratio the delta wye transformer yields a 2 times voltage increase that is useful for PV converters as it allows operation over a wide voltage range with minimal stress on the active devices. As only uni-directional power is required there is no need for active devices on the secondary side and the complexity of a dual active bridge is avoided.

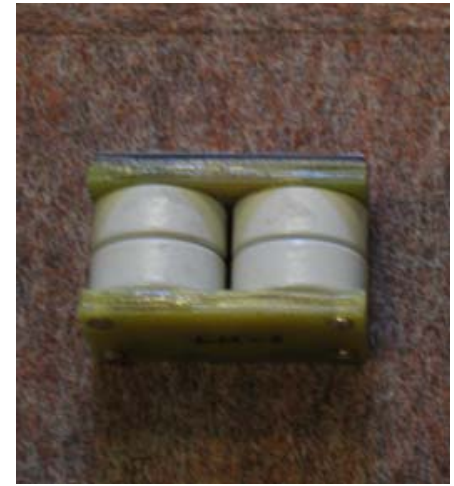

(a) HFCT over view

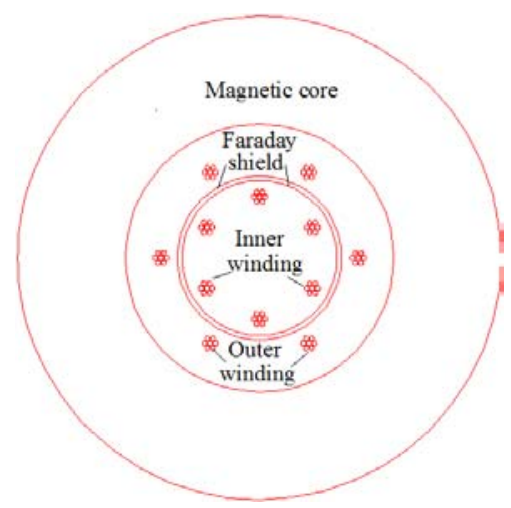

(b) HFCT winding structure of half cross-section

Fig. 2 Configuration of $1 \mathrm{~kW}$ HFCT with shield at operating frequency above $100 \mathrm{kHz}$

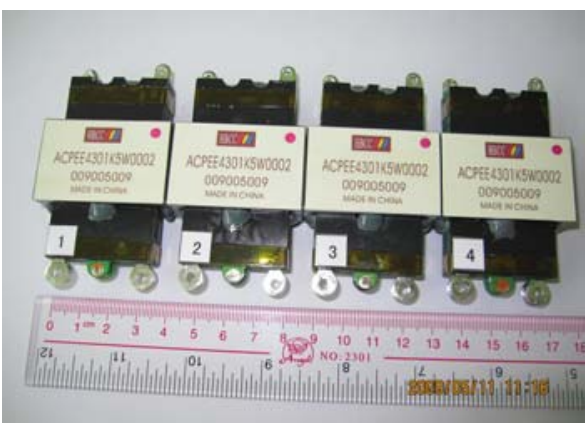

(a) Planar transformer over view

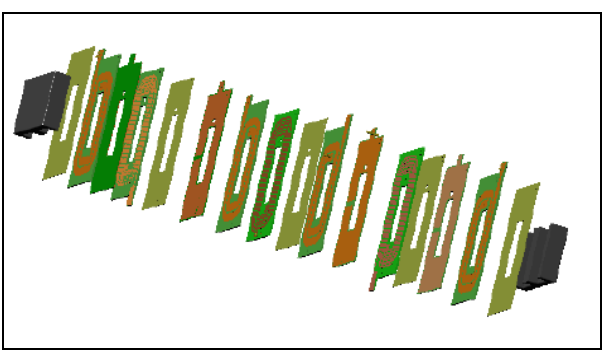

(b) Planar transformer winding structure

Fig.3 Configuration of $1.5 \mathrm{~kW}$ Planar transformer with shield at operating frequency above $100 \mathrm{kHz}$.

\section{Magnetic Field SimUlation AND ANALYSiS}

\section{A. Magnetic field analysis using FEM}

A Finite Element Method (FEM) frequency domain based numerical modelling technique was employed to analyze the magnetic field and eddy current distributions for different winding configurations. The nonlinear magnetic field can be presented by the following equation,

$$
\nabla \times \nu \nabla \times A+\sigma(\partial A / \partial t+\nabla \varphi)-J=0
$$

where $\boldsymbol{A}$ is magnetic vector potential, $\boldsymbol{B}$ represents flux density, $\boldsymbol{v}$ and $\boldsymbol{\sigma}$ are reluctivity and conductivity respectively, $\boldsymbol{J}$ is exciting current.

The detailed magnetic field properties for the high frequency magnetics were then used to facilitate the design of a low loss winding and high efficiency structure. The mathematical problem can be formulated as a sinusoidal quasi-static eddy current problem and is derived from Maxwell's equations. Figures 4 and 5 visualize that the eddy current, induced from the one winding to the other, is smaller with the inserted Faraday shield. As it can be seen in Fig. 4(b) and 5(b), the current distribution with shielding is slightly higher than without shielding, for the simulation results focusing to the primary winding and the secondary windings are under open circuit conditions.

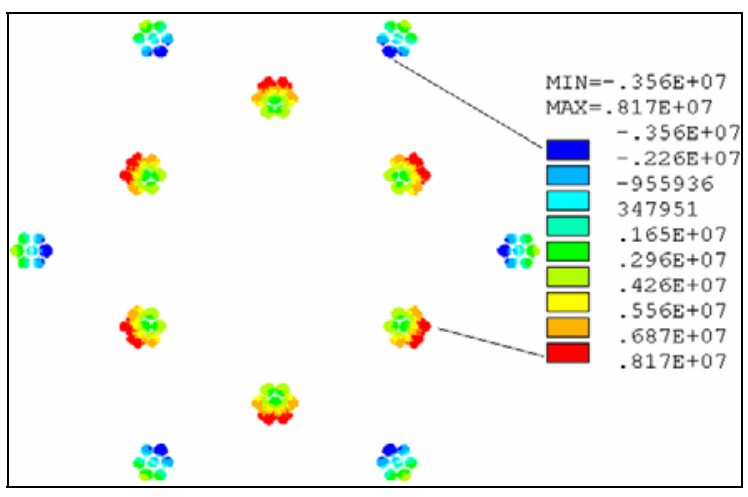

(a) Primary inside without shield 


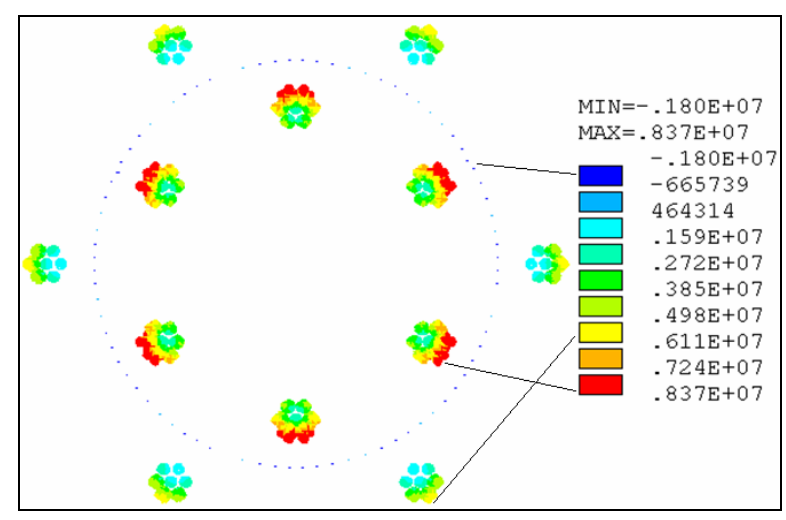

(b) Primary inside with shield

Fig.4 Current distribution in different winding configurations under open circuit conditions.

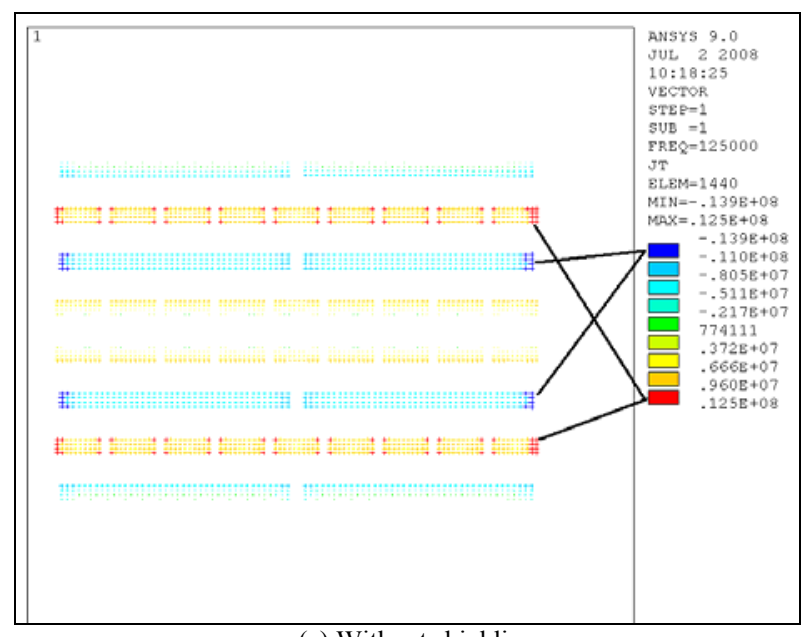

(a) Without shielding

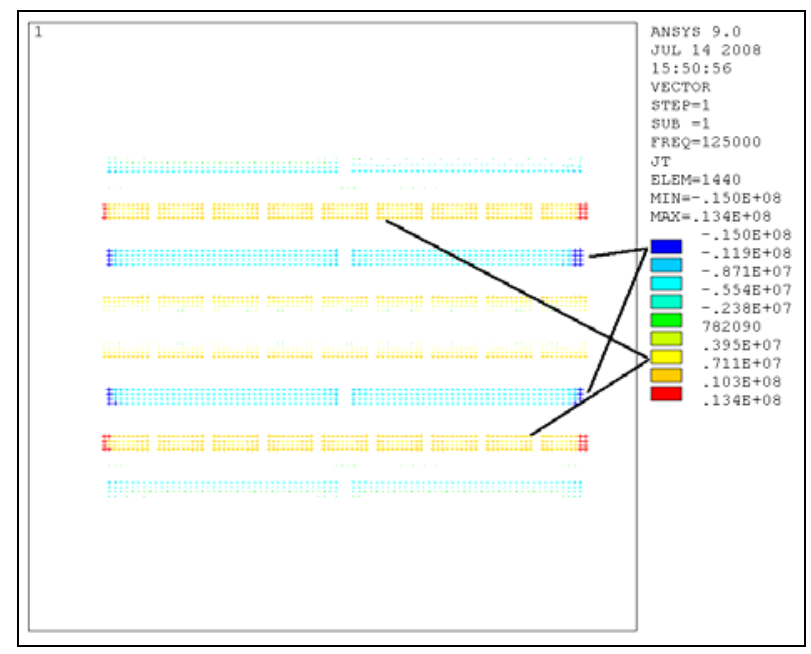

(b) With shielding

Fig.5 Eddy current distribution without shielding and with shielding.

\section{B. Capacitance network model}

The high frequency (HF) noise voltage $\boldsymbol{v}_{\mathrm{s}}$ can be easily propagated to the secondary winding through the coupling capacitance in the no shield case, as shown in Fig. 6(a), is given by:

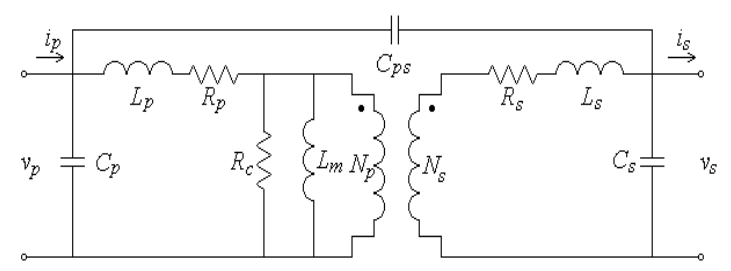

(a) without shield

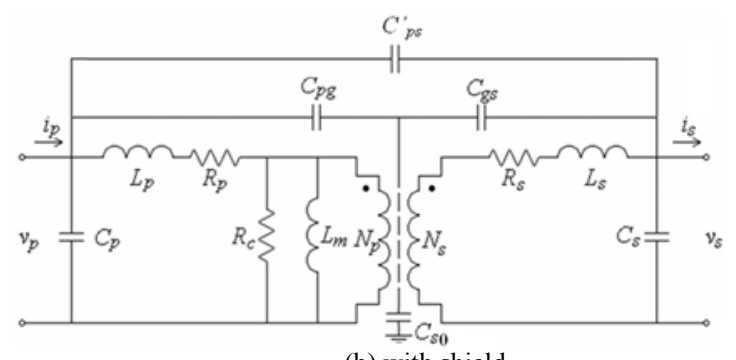

(b) with shield

Fig.6 The HF transformer equivalent circuit.

$v_{\mathrm{s}}=v_{\mathrm{p}} C_{\mathrm{ps}} /\left(C_{\mathrm{ps}}+C_{\mathrm{s}}\right)$

In the perfectly grounded shield case, HF noise voltage $\boldsymbol{v}_{\mathrm{s}}$ is then given by (as shown in Fig. 6(b),)

$v_{s}=v_{\mathrm{p}} C_{\mathrm{ps}}^{\prime} /\left(C_{\mathrm{ps}}^{\prime}+C_{\mathrm{pg}}+C_{\mathrm{s}}\right)$

and for the shield in the floating case, HF noise voltage is given by:

$$
v_{s}=\frac{C_{\mathrm{pg}} \cdot C_{\mathrm{gs}}}{\left(C_{\mathrm{gs}}+C_{\mathrm{s}}\right)\left(C_{\mathrm{pg}}+C_{s 0}\right)+C_{\mathrm{gs}} C_{\mathrm{s}}} v_{p}
$$

The single capacitances in Fig. 7(a) create coupling and therefore HF impacts between the primary and the secondary winding. After insertion of the faraday shield, the HF noise between the windings are strongly dropped, as it can be seen in Fig. 7(b).

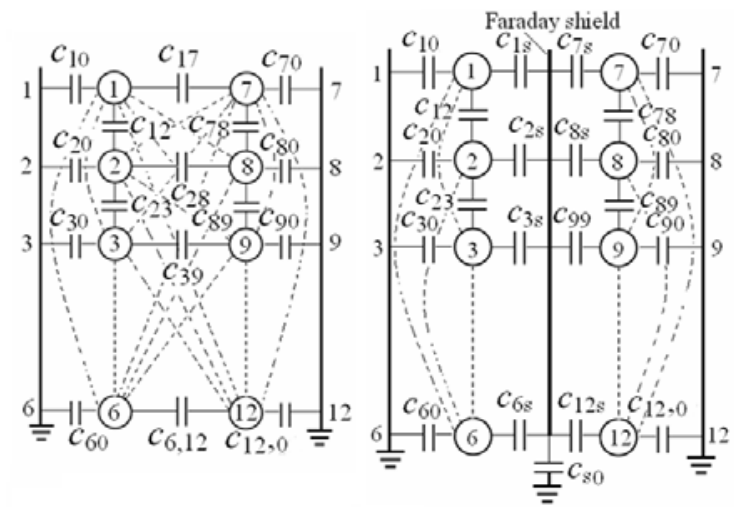

(a) Without Faraday shield,

(b) With Faraday shield.

Fig.7 The parasitic capacitance network model of $\mathrm{HF}$ coaxial transformer for HF noise voltage analysis.

For HF noise analysis of the HFCT, the shield or each turn of the winding can be taken as an independent conductor. Based on the theory of capacitances in multiconductor systems, we can obtain the following set of $N$ 
equations relating the potentials $V_{1}, V_{2}, \ldots, V_{\mathrm{N}}$ of the $N$ conductors to the charges $Q_{1}, Q_{2}, \ldots, Q_{\mathrm{N}}$ :

$$
\left.\begin{array}{l}
Q_{1}=C_{11} V_{1}+C_{12} V_{2}+\cdots+C_{1 \mathrm{~N}} V_{\mathrm{N}} \\
Q_{2}=C_{21} V_{1}+C_{22} V_{2}+\cdots+C_{2 \mathrm{~N}} V_{\mathrm{N}} \\
\vdots \\
Q_{\mathrm{N}}=C_{\mathrm{N} 1} V_{1}+C_{\mathrm{N} 2} V_{2}+\cdots+C_{\mathrm{NN}} V_{\mathrm{N}}
\end{array}\right\}
$$

where the $C_{i i}$ coefficients are called the coefficients of capacitance, and the capacitances $C_{i j}(i \neq j)$ are referred to as the coefficients of induction. The condition of reciprocity, assuming isotropy and linearity, guarantees that $C_{i j}=C_{j i}$. The relation between potential and charge in a multi-conductor system can be described by the electric scalar potential $\boldsymbol{V}$, which satisfies Poisson's equation,

$$
-\nabla \cdot(\varepsilon \nabla V)=\rho
$$

where $\varepsilon$ is the permittivity, and $\rho$ is the space charge density.

To obtain the capacitance from Eq. (6), the charge density must be calculated using a FEM analysis. The 2D FEM system matrix equation can be expressed as (8) for the relationship between charge and potential,

$$
[S]\{V\}=\{Q\}
$$

where $[\mathrm{S}]$ is the global coefficient matrix and $\{Q\}$ is the charge matrix. By setting up the boundary condition, $V_{1}=1, V_{2}=V_{3}=\ldots=V_{\mathrm{N}}=0$, we can obtain $Q_{1}=C_{11}, Q_{2}=C_{21}$, $\ldots, Q_{\mathrm{N}}=C_{\mathrm{N} 1}$, and in the same manner, the coefficients $C_{i i}$ 's and $C_{i j}$ 's can be calculated.

From the $C_{i i}$ and $C_{i j}$ coefficients, the capacitance of the $i^{\text {th }}$ conductor to ground, $c_{i 0}$, and the capacitance between the $i^{\text {th }}$ conductor and the $j^{\text {th }}$ conductor, $c_{i j}(i \neq j)$, can be calculated. Thus, all of the parasitic capacitances in the network model shown in Fig.7 can be obtained.

The calculated results show that the inter- winding capacitance is reduced significantly from $19.96 \mathrm{pF}$ to $0.08 \mathrm{pF}$ due to the insertion of a Faraday shield in the HFCT. While, the intra- winding capacitance $C_{\mathrm{p}}$ increases from $7.67 \mathrm{pF}$ to $24.67 \mathrm{pF}$ and $C_{s}$ increases from $3.55 \mathrm{pF}$ to $22.50 \mathrm{pF}$, because $C_{\mathrm{pg}}$ and $C_{\mathrm{gs}}$ are added to the primary and secondary sides respectively through the ground, as shown in Fig. 7(b). The capacitance $C_{\mathrm{s} 0}$ does not exist if the shield is perfectly grounded. It is obvious that by integrating a Faraday shield into the HFCT, the value of $C_{\mathrm{ps}}$ is decreased, and at the same time the value of $C_{\mathrm{s}}$ is increased. Both the decreased value for $C_{\mathrm{ps}}$ and the increased value for $C_{s}$ will improve the noise suppression and reduce the impact of transients on semiconductor devices [6]. The measured value for the prototype HFCT is $21.7 \mathrm{pF}$, which is in good agreement with the calculated value of $19.96 \mathrm{pF}$.

From (3), (4), and (5), the EMI noise reduction can be calculated for the aforementioned three cases, respectively. TABLE I confirms that a perfectly grounded shield can provide the best EMI suppression. The EMI reduction is down to $-49.90 \mathrm{db}[7]$.

TABLE I

Times New Roman Type Sizes AND STyLes

\begin{tabular}{|l|l|l|l|}
\hline & No shield & $\begin{array}{l}\text { Shield } \\
\text { grounded }\end{array}$ & $\begin{array}{l}\text { Shield } \\
\text { floating }\end{array}$ \\
\hline $\begin{array}{l}\text { Voltage } \\
\text { ratio }\end{array}$ & 0.849 & 0.0032 & 0.473 \\
\hline $\begin{array}{l}\text { EMI } \\
\text { reduction } \\
(\mathrm{db})\end{array}$ & -1.42 & $\mathbf{- 4 9 . 9 0}$ & -6.50 \\
\hline
\end{tabular}

\section{CONCLUSIONS}

A detailed discussion of a high frequency coaxial and planar isolation transformers used for a solar PV DC/DC converter is presented. A FEM eddy current simulation is used to investigate the winding configurations and Faraday shield arrangements under different load conditions. The results show that the extra peak current density and the extra power loss caused by the insertion of Faraday shield can be neglected and the magnetic flux distribution is not influenced by the insertion. A parasitic capacitance network model is proposed to calculate these capacitances by FEM for a HFCT with or without a Faraday shield. The calculated inter-capacitance value for the HFCT without shield is in good agreement with the experimental result. The presented approach for calculating intra- and inter- capacitances of HFCT can be extended to the calculation of the intra- and intercapacitance for other high frequency transformers.

\section{ACKNOWLEDGMENT}

The authors would like to thank Dr. X. Yang for his helpful discussion and the assist on magnetic field simulation.

\section{REFERENCES}

[1] M. H. Kheraluwala, D. W. Novotny, D. M. Divan, "Coaxially Wound Transformers for High-Power HighFrequency Applications," IEEE Trans. On Power Electronics, Vol. 7 No. 1, pp. 54-62, Jan. 1992.

[2] J. Lu, F. Dawson, and S. Yamada, "Analysis of high frequency planar sandwich transformers for switching converters," IEEE Trans. Magn., vol. 31, pp. 4235-4237, Nov. 1995.

[3] K.W. Klontz, D. W. Novotny and et.al, "An active cooled $120 \mathrm{~kW}$ coaxial winding transformer for fast charging electric vehicles," IEEE Trans. on Industry Applications, vol. 31, No. 6, pp. 1257-1263, Nov/Dec. 1995.

[4] J. Lu, F. P. Dawson and S. Yamada, "Application and analysis of adjustable profile high frequency switch mode transformer having a U-shaped winding structure", IEEE Trans. on Magnetics, Vol. 34. No. 4, pp. 3186-3188, July 1998.

[5] David K. Chen, Field and Wave Electromagnetics, Second Edition, Addison Wesley, 1989, pp.129-132.

[6] L.Tihanyi, Electromagnetic Compatibility in Power Electronics. Piscataway, NY: IEEE Press, 1995, pp.143-146.

[7] S. Stegen and J. Lu, "Shielding effect of High Frequency Power Transformers for DC/DC Converter used in Solar PV Systems", Proceedings of APEMC2010, Beijing, April, 2010 